\title{
Giant cell tumour of bone:
}

\section{A demographic study from a tumour unit in South Africa}

\author{
Dr A Zanati MBChB, HDip Orth(SA) \\ Dr N Ferreira BSc, MBChB, HDip Orth(SA), FC Orth(SA), MMed(Orth) \\ Dr LC Marais MBChB, FCS Orth(SA), MMed(Ortho), CIME, PhD
}

Tumour Sepsis and Reconstruction Unit, Department of Orthopaedic Surgery, Grey's Hospital, Nelson R Mandela

School of Medicine, University of KwaZulu-Natal

\author{
Corresponding author: \\ Dr Nando Ferreira \\ Tumour, Sepsis and Reconstruction Unit \\ Department of Orthopaedic Surgery \\ Grey's Hospital \\ Nelson R Mandela School of Medicine \\ University of KwaZulu-Natal \\ 3201 Pietermaritzburg, South Africa \\ Tel: +27338973000 \\ Email: drferreiran@gmail.com
}

\begin{abstract}
Introduction:

Giant cell tumour of bone (GCT) is a rare primary bone tumour. Little is known about the epidemiology of this tumour in South Africa as most demographic information is based on research from Asia, Europe and North America. This research aims to raise awareness and promote early recognition of these tumours.

Materials and methods:

A retrospective analysis was conducted of all patients with biopsy-confirmed GCTs that presented between January 2010 and December 2014. Information pertaining to patient demographics, tumour location, treatment and outcome was recorded and analysed.

Results:

Twenty-two patients were included in the study. The mean age of patients was 32.4 years (range 12-63), and a slight male predominance (1.2:1) was observed. Tumours were mainly located at the end of long bones $(91 \%)$ with the distal femur and proximal tibia being most commonly affected (55\%). Two patients (9\%) were diagnosed with primary malignant giant cell tumours. We observed a higher rate of lung metastases (18\%) than previously reported. The median tumour volume was significantly higher in patients who developed lung metastases $\left(467.4 \mathrm{~cm}^{3}\right.$ vs $\left.137.8 \mathrm{~cm}^{3} ; \mathrm{p}=0.03\right)$. Three of the patients with lung metastases were HIV-positive (odds ratio [OR] = $10.5,95 \%$ confidence interval $[\mathrm{CI}]=0.84-130.66, \mathrm{p}=0.076$ ). All patients were treated surgically with extended curettage, local adjuvant therapy, polymethyl methacrylate (PMMA) and internal fixation or en-bloc resection with prosthetic or osteochondral allograft replacement.
\end{abstract}

\section{Conclusion:}

Giant cell tumours of bone are uncommon. Demographics from South Africa emulate international statistics. No recurrence of GCTs was observed in our cohort despite the relatively large tumours at time of presentation compared to international literature that report recurrence rates of approximately $2 \%$. The incidence of metastases and primary malignant GCT was higher than in previous reports. The association of these findings with HIV infection warrants further investigation. Metastases appear to be associated with the size of the primary tumour.

Key words: giant cell tumour of bone, demography, benign tumour 


\section{Introduction}

Giant cell tumour of bone (GCT) is a relatively uncommon primary bone tumour. ${ }^{1}$ It accounts for $5-6 \%$ of all primary bone tumours and approximately $20 \%$ of benign bone tumours. Variations in incidence have been reported from around the word. ${ }^{2-4}$ Significantly higher incidence rates have been observed in China and southern India, where these tumours constitute about $20 \%$ of all primary bone tumours. ${ }^{5}$

GCTs are benign but locally aggressive mesenchymal neoplasms with unpredictable biological behavior. ${ }^{6}$ The majority of cases $(80 \%)$ occur between the third and fifth decade of life and a slight female predominance with a male-to-female ratio of 1:1.5 has been reported. ${ }^{2,3}$ Less than $3 \%$ of cases occur before the age of 14 years, and only 13\% of cases occur in patients over the age of 50 years. ${ }^{5}$ These tumours are typically located at the ends of long bones (distal femur, proximal tibia, distal radius and proximal humerus) but have been reported in almost all other anatomical sites. ${ }^{6,7}$ Patients usually present with swelling and activity-related pain that can progress to pain at rest. In rare occasions patients may remain relatively asymptomatic until they develop a pathological fracture. ${ }^{1,6}$

The diagnosis of GCT is based on radiographic imaging in conjunction with confirmatory histology. Standard radiographs exhibit lesions that are usually eccentrically located in the epiphysis, extending into the metaphysis, of long bones. These lesions appear purely lytic and are locally destructive. The centre of the lesion sometimes has a soap-bubble appearance due to ridging of the surrounding bone. There is typically no clear zone of transition, no marginal sclerosis and no periosteal reaction..$^{1,5,6}$ Magnetic resonance imaging (MRI) findings include heterogenous high T2 signal with interspersed low signal areas or fluid-fluid levels in case of secondary aneurysmal bone cyst formation. MRI provides useful information regarding involvement of the adjacent joint and the extent of the lesion within the bone and soft tissue. ${ }^{8}$ Computerised tomography (CT) scans may add further information regarding the extent of the tumour but are not typically used. A bone scintigraphy scan is helpful if multi-centric tumours are suspected.

Histologically, GCTs are characterised by numerous multinucleated osteoclast-like giant cells that are scattered in a background of homogenous mononuclear stromal cells. ${ }^{1}$ The mononucleated cells are composed of two distinct cellular components, a spindle-shaped major component and a minor component consisting of monocyte-derived macrophages. The spindle-like stromal cells are of the osteoblast lineage and form the main neoplastic component of these tumours. These cells play a central role in bone destruction through the production of receptor activator of NF- $\kappa B$ ligand (RANKL) that binds to osteoclasts and induces osteolysis. ${ }^{9}$ Secondary aneurysmal bone cyst formation may also be present. ${ }^{8}$ GCT has the ability to metastasise although this does not necessarily herald malignant transformation. These benign metastases are usually to the lungs and have been reported to occur in $2 \%$ of cases. ${ }^{1,6}$ Malignant change, on the other hand, is defined as sarcomatous change in the primary lesion. ${ }^{10}$ This malignant transformation is rare and only occurs in approximately $1 \%$ of cases. ${ }^{11-13}$ Primary malignancy in GCT refers to the synchronous coexistence of a sarcoma and benign GCT within a lesion while secondary malignancy describes a sarcomatous growth in a previously treated, biopsy-confirmed, benign GCT. ${ }^{14,15}$

The treatment of GCTs has not changed much in the past 30 years. This is in part due to the relative rarity of the tumours and the lack of randomised clinical trials. ${ }^{4}$ Surgery represents the mainstay of treatment. This usually involves extended curettage with a high-speed burr and chemical adjuvants in the form of either liquid nitrogen or phenol followed by filling of the tumour cavity with PMMA bone cement. ${ }^{1,5}$ Patients with unresectable tumours are treated with external beam radiation but transformations to malignant sarcoma have been reported. ${ }^{16}$ Recently, new chemotherapeutic drugs like Denosumab (monoclonal antibody) have successfully been used in the management of GCTs. ${ }^{17}$

This retrospective review aims to establish the first demographic data for GCTs in South Africa.

\section{Materials and methods}

We retrospectively reviewed a cohort of consecutive patients, seen between January 2010 and December 2014, who were diagnosed with giant cell tumour of bone. All cases of biopsy-confirmed GCT were included in the evaluation. Patients charts were reviewed and data extracted pertaining to patient demographics, tumour location, treatment and outcome.

Institutional ethics committee approval was obtained prior to commencement of the study.

\section{Management protocol}

All patients were admitted for local and systemic staging. Local staging consisted of radiographs and an MRI scan. Systemic staging included work-up for medical comorbidities, laboratory investigations and a CT scan of the chest. Tumour volume was calculated based on MRI images using the formula for an ellipsoidal tumour mass, where volume $=(\pi / 6) \times$ length $\times$ width $\times$ height. Histology was obtained by formal incisional biopsy, according to standard biopsy principles, in all cases. Diagnosis was subsequently confirmed by combined radiological and histological evaluation. Definitive treatment involved either extended curettage or en-bloc resection. Extended intralesional excision (incorporating the use of a high-speed burr to resect the adjacent bony margins) in conjunction with local adjuvant (80\% phenol or liquid nitrogen), PMMA and locked plating was performed in all cases without pathological fracture and/or intra-articular extension. In cases where concern existed about a break in the continuity of the 
subchondral bone and cartilage, liquid nitrogen was preferred above phenol as local adjuvant therapy due to the risk of intra-articular spillage. Cases involving pathological fracture with significant soft tissue extension, intra-articular extension or loss of structural integrity that was not considered reconstructable with PMMA and locked plating were treated with en-bloc resection and modular endoprosthesis (GMRS, Stryker) or osteoarticular allograft replacement. Tumours with histological evidence of malignancy were treated by wide excision and modular endoprosthetic replacement. Patients were followed up, clinically and radiologically, three monthly during the first two years postoperatively and then six monthly.

\section{Statistical analysis}

Statistical analysis was performed using Stata 13.0 (StataCorp. College Station, Texas). Differences in continuous variables were compared with the use of the Wilcoxon rank-sum test. Categorical variables (metastases and malignancy) were cross-tabulated against HIV status and the association was analysed using the Fisher exact probability test. All tests were two-sided and the level of significance was set at $\mathrm{p} \leq 0.05$.

\section{Results}

Twenty-four patients met the inclusion criteria. Two patients were excluded. The first patient had polyostotic lesions with a histological diagnosis of a GCT. This patient is however being further investigated for suspected hyperparathyroidism. The second patient had an initial histological diagnosis of GCT of bone but confirmatory histology after definitive surgery showed a chondroblastoma with a minor aneurysmal bone cyst component.

The final cohort consisted of 22 patients with a mean age of 32.4 years (range 12-63) (Table I). The majority of patients $(72.7 \%)$ were in the third and fourth decades of life. (Figure 1) A slight male predominance with a male-to-female ratio of 1.2:1 (12 men and 10 women) was observed. Seven (31.8\%) patients were HIV positive with a mean CD4 count of $265.8 \mathrm{cell} / \mathrm{cm}^{3}$ (range 59-454). Six HIV-positive patients were on highly active antiretroviral therapy. Patients with CD4 counts below 350 cells $/ \mathrm{cm}^{3}$ were started on antiretroviral therapy prior to surgery.

The majority of giant cell tumours arose around the knee with nine out of $22(40.9 \%)$ involving the proximal tibia and three (13.6\%) involving the distal femur (Figures 2 and 3).

\begin{tabular}{|c|c|c|c|c|c|c|}
\hline Patient & Age (yr) & Gender & HIV status & Anatomical location & Metastases & Histological diagnosis \\
\hline 1 & 36 & Male & Positive & Proximal tibia & Lung mets & Malignant GCT \\
\hline 2 & 31 & Female & Negative & Proximal tibia & None & GCT/Secondary ABC \\
\hline 3 & 22 & Male & Positive & Proximal tibia & Lung mets & GCT \\
\hline 4 & 28 & Male & Positive & Proximal tibia & None & GCT \\
\hline 5 & 22 & Male & Negative & Proximal tibia & None & GCT \\
\hline 6 & 25 & Female & Negative & Proximal tibia & None & GCT \\
\hline 7 & 36 & Male & Positive & Proximal tibia & Lung mets & GCT \\
\hline 8 & 23 & Male & Negative & Proximal tibia & None & GCT \\
\hline 9 & 35 & Female & Positive & Proximal tibia & None & Atypical GCT \\
\hline 10 & 29 & Female & Positive & Distal femur & None & GCT \\
\hline 11 & 38 & Male & Negative & Distal femur & None & GCT \\
\hline 12 & 46 & Male & Negative & Distal femur & None & GCT \\
\hline 13 & 27 & Male & Positive & Proximal femur & None & GCT \\
\hline 14 & 26 & Male & Negative & Proximal femur & None & GCT \\
\hline 15 & 55 & Male & Negative & Proximal femur & Lung mets & GCT \\
\hline 16 & 34 & Female & Negative & Proximal femur & None & GCT \\
\hline 17 & 12 & Male & Negative & Distal humerus & None & GCT \\
\hline 18 & 30 & Female & Negative & Distal radius & None & GCT \\
\hline 19 & 23 & Female & Negative & Distal radius & None & GCT \\
\hline 20 & 63 & Female & Negative & Distal ulna & None & Malignant GCT \\
\hline 21 & 50 & Female & Negative & Pelvis (pubic ramus) & None & GCT/Secondary ABC \\
\hline 22 & 16 & Female & Negative & Pelvis (iliac bone) & None & GCT \\
\hline
\end{tabular}




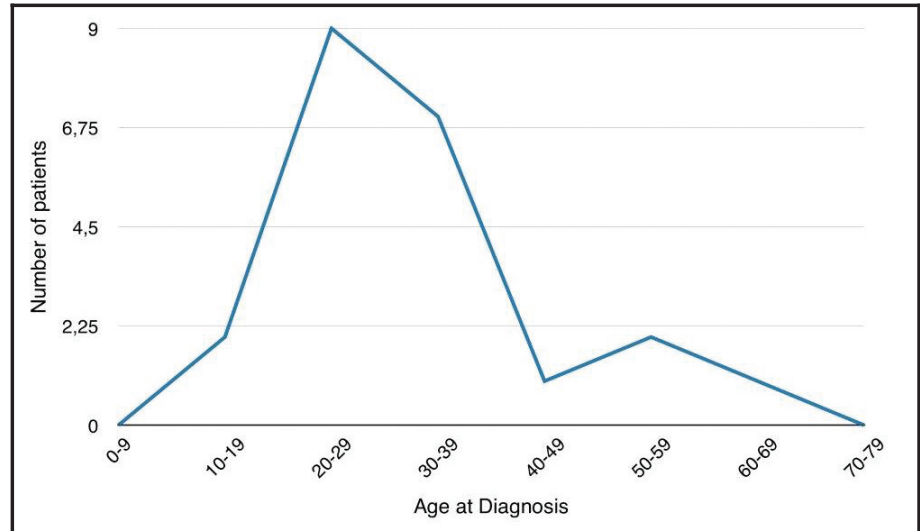

Figure 1. Age distribution

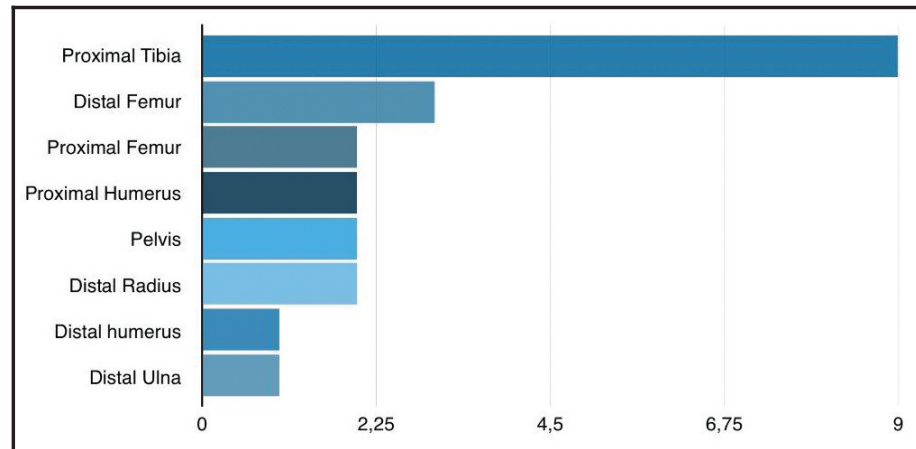

Figure 2. Anatomical locations

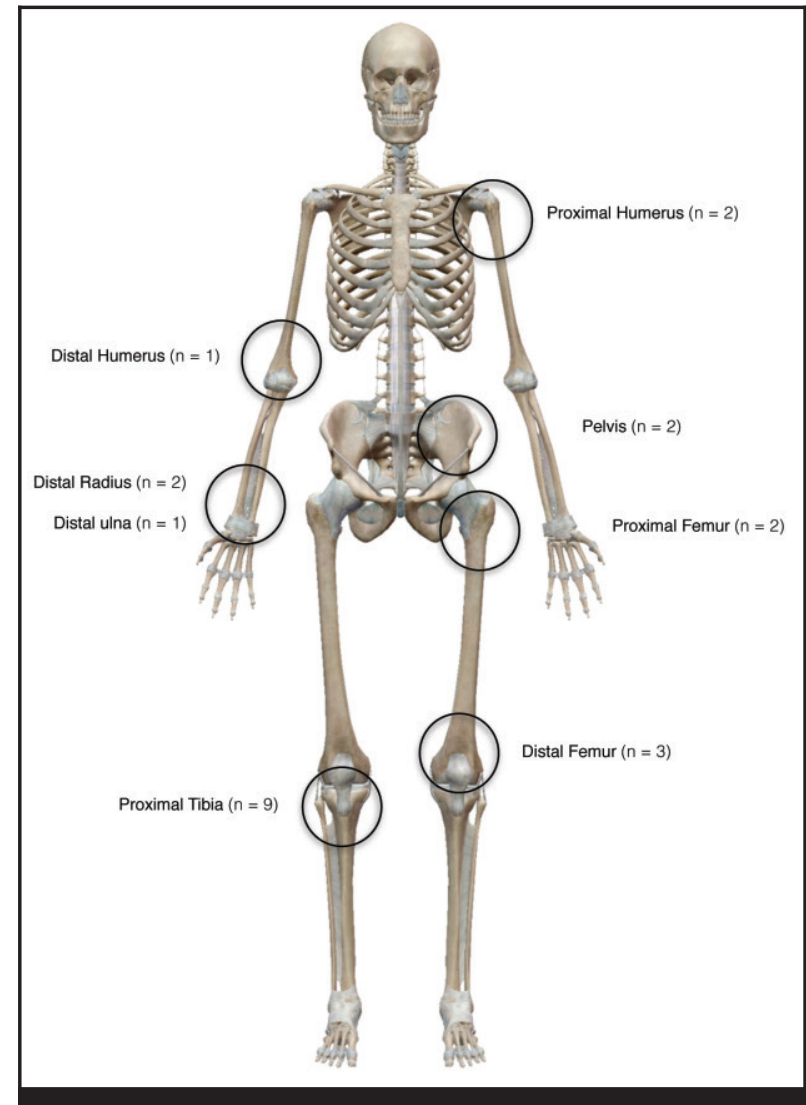

Figure 3: Giant cell tumour of bone anatomical distribution
The remaining tumours arose from the proximal femur $(\mathrm{n}=2)$, proximal humerus $(\mathrm{n}=2)$, distal radius $(\mathrm{n}=2)$, pelvis $(n=2)$, distal humerus $(n=1)$ and distal ulna $(n=1)$. Average tumour volume was $201.7 \mathrm{~cm}^{3}$ ranging from $15.6 \mathrm{~cm}^{3}$ to $3138 \mathrm{~cm}^{3}$.

Two patients $(9.1 \%)$ were diagnosed with primary malignant GCTs; the first involved the proximal tibia while the second was located in the distal ulna. Four patients $(18.2 \%)$ presented with lung metastases. Three patients had histologically confirmed benign GCTs while the remaining patient was diagnosed with a primary malignant GCT of the proximal tibia. The median tumour volume for patients with lung metastases was $467.4 \mathrm{~cm}^{3}$ (range $160.4 \mathrm{~cm}^{3}-3 \quad 138 \mathrm{~cm}^{3}$ ) compared to a median tumour volume of $137.8 \mathrm{~cm}^{3}$ (range $15.6 \mathrm{~cm}^{3}-679.3 \mathrm{~cm}^{3}$ ) for patients without lung metastases $(\mathrm{p}=0.03)$. There was no significant difference in the size of the tumours according to HIV status $(\mathrm{p}=0.55)$. Three of the patients with lung metastases were HIV-positive (odds ratio $[\mathrm{OR}]=10.5,95 \%$ confidence interval $[\mathrm{CI}]=0.84-130.66, \mathrm{p}=0.076$ ). We found no association between the presence of metastases and the age of the patient $(\mathrm{p}=0.4)$.

All patients were treated surgically. Ten patients underwent tumour resection and megaprosthesis reconstruction. Four $(40 \%)$ of these patients were HIVpositive. Megaprosthesis reconstruction was performed for seven tumours that were located around the knee, two proximal femurs and one proximal humerus. Six patients were treated with extended curettage with liquid nitrogen followed by PMMA bone cement and locking plate fixation. Two patients with GCTs involving the distal radius were treated by resection of distal radius followed by size matched allograft replacement and locking plate fixation. The tumour located in the ulna was treated by wide resection of the distal ulna without reconstruction. One tumour that was located in the superior pubic ramus was treated with bone graft following the extended curettage. Two patients required amputation as the initial management in order to achieve pain control and limit impairment. Both of these were massive tumours that were located in the proximal tibia and unresectable at time of presentation.

Average follow-up was 21 months and ranged from two to 54 months. One patient died during the follow-up period. This male patient was HIV-positive and presented with an unresectable tumour in the proximal tibia and lung metastases. No tumour recurrence was observed during the follow-up period.

\section{Discussion}

The current study documents the demographic information, tumour location, treatment and outcome of 22 patients diagnosed, clinically and histologically, as GCT of bone. 
We observed a slight male predominance (1.2:1), which is contradictory to international literature that reports a female predominance of $1: 1.5$. $^{2,3,8}$ Our results reflect international literature with $81 \%$ of cases being between the ages of 20 and 50 years. ${ }^{2,3}$ Less than $5 \%$ of cases involved children younger than 14 years of age, and $9 \%$ of patients were older than 50 years. ${ }^{5}$

GCTs are typically located at the ends of long bones. . $^{5-7}$ The distal femur and proximal tibia are involved in $50 \%$ to $65 \%$ of cases, with the distal radius being affected in about $10 \%$ of cases. ${ }^{46}$ The proximal femur and humerus are affected in less than $10 \%$ of cases respectively. ${ }^{18-22}$ GCTs involving the pelvis and ulna are rare. ${ }^{23-25}$ Our results mostly concurred with these reports, with $55 \%$ of tumours occurring around the knee, 9\% in the distal radius and 9\% in the proximal femur. We observed a slightly higher incidence of tumours involving the humerus $(14 \%)$, ulna $(4 \%)$ and pelvis $(9 \%)$, than previously reported.

Fluid-fluid levels, consistent with secondary formation of aneurysmal bone cysts (ABC), have been reported in up to $14 \%$ of GCT cases. ${ }^{5}$ We observed secondary $\mathrm{ABC}$ formation in two tumours (9\%).

Benign GCTs have the ability to metastasise and this usually involves spread to the lungs. The overall risk for metastatic spread ranges from $1 \%$ to $9 \%$, with the largest series reporting a rate of $2.6 \% .^{26,27}$ Mortality from lung metastases in GCTs range from $14 \%$ to $23 \% .^{26,28,29}$ We observed lung metastases in $15 \%$ of benign GCTs. The median tumour volume was significantly higher in patients who developed lung metastases $\left(467.4 \mathrm{~cm}^{3}\right.$ vs $\left.137.8 \mathrm{~cm}^{3} ; \mathrm{p}=0.03\right)$. Three of these patients $(75 \%)$ were HIVpositive, but this was found not to be statistically significant $(p=0.07)$. The mortality rate of GCT with lung metastases in our series was $25 \%$.

Primary malignancy in GCT accounts for less than $1 \%$ of cases in international reports. ${ }^{14}$ We observed two cases $(9 \%)$ of primary malignancy. One occurred in the proximal tibia of a HIV-positive male and the other in the distal ulna of a HIV-negative female ( $\mathrm{p}=0.54$ ).

The recurrence rate after extended curettage with highspeed burr and liquid nitrogen or phenol is reported to be as low as $2 \% .{ }^{1,5}$ No recurrence was observed in this series with the use of extended curettage in combination with either cementing or bone grafting of the defect.

This study has several shortcomings. The retrospective nature of the analysis and the small number of cases prevents drawing definitive conclusions. This report does however provide novel information about the demographics of GCTs in the South African clinical setting and in HIV-positive patients. The increased rate of metastases is an interesting finding and the relationship with the size, duration or Campanacci classification of the primary tumour needs further investigation. Although there was a trend towards increased rate of metastases in HIV-positive patients, this did not reach statistical significance and larger series of cases will be required.

\section{Conclusion}

This retrospective review presents the demographic results and treatment outcome of 22 patients with GCT diagnosed and treated in a higher institution. We observed a slight male predominance and the majority of patients were in the third and fourth decades of life. The anatomical distribution of tumours was also consistent with previous reports. The incidence of metastases (15\%) and primary malignant GCT (9\%) is higher than in other reports. The association between these findings and HIV infection warrants further investigation. The occurrence of lung metastases appears to be associated with the size of the primary tumour.

\section{Compliance with ethics guidelines}

Institutional ethics committee approval was obtained prior to commencement of the study.

Drs Zanati, Ferreira and Marais declare that the content of this article is their original work. No benefits of any form have or will be received from any commercial party related directly or indirectly to the subject of this article.

\section{References}

1. Apley AG, Solomon L. Apley's system of orthopaedics and fractures. 7th ed. Butterworth-Heimann. 1999;175-76.

2. Gupta R, Seethalakshmi V, Jambhekar NA, Prabhudesai S, Merchant N, Puri A, et al. Clinicopathologic profile of 470 giant cell tumours of bone from a cancer hospital in western India. Ann Diagn Pathol 2008;12:239-48.

3. Unni KK, Inwards C. Dahlin's Bone tumours: general aspects and data on 10,165 cases. 6th Edition. Philadelphia, PA: Lippincott Williams \& Wilkins 2010;225-42.

4. Thomas DM, Skubitz T. Giant-cell tumour of bone. Current Opinion in Oncology 2009;21:338-44.

5. Chakarun CJ, Forrester DM, Gottsegen CJ, Patel DB, White EA, Matcuk GR. Giant Cell Tumor of Bone: Review, Mimics, and New Developments in Treatment. Radiographics 2013;33:197-211.

6. Miller MD. Review of Orthopaedics. 6th ed. Philadelphia, PA: Saunders. 2004;485-87.

7. Hoch B, Hermann G, Klein MJ, Abdelwahab IF, Springfield D. Giant cell tumour complicating Paget disease of long bone. Skeletal Radiol 2007;36(10):973-78.

8. Campbell's Operative Orthopaedics, 11th ed. Mosby, An Imprint Elsevier; 2007:883-85.

9. Steensma MR, Tyler WK, Shaber AG, Goldring SR, Ross $\mathrm{FP}$, Williams BO, et al. Targeting the giant cell tumour stromal cell: Functional characterization and a novel therapeutic strategy. PloS ONE. 2013 Jul;8(7):e69101.

10. Hunter RV, Worcester J, Francis KC, Foote FW, Stewart FW. Benign and malignant giant cell tumors of bone. A clinicopathological analysis of the natural history of the disease. Cancer 1962;15:653-90.

11. Brien EW, Mirra JM, Kessler S, Suen M, Ho JK, et al. Benign giant cell tumour of bone with osteosarcomatous transformation ('dedifferentiated' primary malignant GCT): report of two cases. Skeletal Radiol.1997;26:246-55. 
12. Gulhane SR, Kate MS. Sarcomatous transformation in a conventional giant cell tumour of femur. NJIRM 2011;2:124-26.

13. Sakkers RJ, van der Heul RO, Kroon HM, Taminiau AH, Hogendoorn PC. Late malignant transformation of a benign giant-cell tumor of bone. A case report. J Bone Joint Surg Am.1997;79:259-62.

14. Junior RZ, de Camargo OP, Ida CM, Baptista AM, Ribeiro MB, Bruno JM, et al. Primary malignancy in giant cell tumour: a case report. Sao Paulo Med J. 2009;127(5):310-13.

15. Ferreira N, Marais LC. Primary malignant giant cell tumour of the proximal tibia: a case report. J Cancer Res Ther. 2013;1(6):174-77.

16. Mendenhall W, Zlotecki R, Scarborough M, Gibbs C, Mendenhall N. Giant Cell tumour of bone. Am J Clin Oncol 2006;29(1):96-99.

17. Thomas D, Henshaw R, Skubitz K, et al. Denosumab in patients with giant-cell tumour of bone: an open-label, phase 2 study. Lancet Oncol 2010;11(3):275-80.

18. Dhatt S, Tahasildar N, Tripathy S, BK S, Tamuk T. Excision and endoprosthesis implantation for proximal femur giant cell tumor. WebmedCentral ORTHOPAEDICS 2010;1(11):WMC001236.

19. Oda $Y$, Miura H, Tsuneyoshi M, et al. Giant cell tumor of bone: oncological and functional results of long-term follow-up. Jpn J Clin Oncol 1998;28:323-28.

20. O'Donnell RJ, SpringWeld DS, Motwani HK, et al. Recurrence of giant-cell tumours of the long bones after curettage and packing with cement. J Bone Joint Surg Am 1994;76:18271-833.

21. Sait SA, Nithyanath M, Cherian VM. Giant cell tumour of the distal humerus treated with elbow arthroplasty: A Case Report. International Journal of Case Reports and Images 2012;3(4):37-40.
22. Stiepan FE. Giant cell tumour of the head of the humerus: A Case Report. J Bone Joint Surg Am 1954 Oct;36(5):101419.

23. Vanni D, Pantalone A, Andreoli E, Caldora P, Salini V. Giant cell tumor of the distal ulna: A case Report. Journal of Medical Case Reports 2012;6:143.

24. Goldenberg RR, Campbell CJ, Bonfiglio M. Giant cell tumor of bone. An analysis of two hundred and eighteen cases. Journal of Bone and Joint Surgery 1970;52(4):619-64.

25. Zheng K, Wang Z, Wu S, Ye Z, Xu S, Xu M, et al. giant cell tumor of the pelvis: a systemic review. Orthopaedic Surgery 2015;7:102-107.

26. Burke CS, Gupta A, Buecker P. Distal ulna giant cell tumor resection with reconstruction distal ulna prosthesis and brachioradialis wrap soft tissue stabilization. Hand (New York)2009 Dec;4(4):410-14.

27. Kay RM, Echardt JJ, Seeger LL, Mirra JM, Hak DJ. Pulmonary metastasis of benign giant cell tumor of bone; six histologically confirmed including of one of spontaneous regression. Clin Orthop Relat Res 1994;302:219-30.

28. Campanacci M, Baldini N, Boriani S, Sudanese A. Giant cell tumor of bone. J Bone Surg. 1987;69A:106-14.

29. Tubbs WS, Brown LR, Beabout JW, Rock MG, Unni KK. Benign giant cell tumor of bone with pulmonary metastases: clinical findings and radiologic appearance of metastases in 13 cases. AJR Am J Roentgenol. 1992;158:331-34.

This article is also available online on the SAOA website (www.saoa.org.za) and the SciELO website (www.scielo.org.za). Follow the directions on the Contents page of this journal to access it. 